\title{
An Analysis of Pedestrian Queueing
}

\author{
George H. Weiss*
}

(June 10, 1963)

\begin{abstract}
This paper considers the steady state queueing properties of pedestrians who are delayed by traffic on a main road. It is assumed that the size of the gap between two successive cars determines the probability that an individual pedestrian or a group of pedestrians will cross. The technique of imbedded Markov chains yields many new results as well as some previously found, by combinatorial methods by Tanner in 1951.
\end{abstract}

\section{Introduction}

There has been a recent increase of interest in the problem of the delay to traffic at a stop sign. This problem was first suggested by data analyzed by Adams in 1936, [1]. ${ }^{1}$ Adams derived an expression for the mean delay to a pedestrian caused by a stream of traffic on a main highway, in which the successive gaps (in time) are independent random variables, each with a negative exponential distribution. It was assumed that each pedestrian waits for a gap which is larger than a prescribed safe interval before attempting to cross. Garwood, in 1941 [2], took up the problem in a modified form. He dealt with the problem of driver delay at an intersection in which a traffic signal is partially operated by the main stream traffic and partially by the crossing traffic. When a crossing car arrives at the intersection it actuates the signal so that the light will change to green either at a fixed time $M$ after its arrival, or at some earlier time if the main stream is sufficiently light. Garwood's analysis assumed a negative exponential distribution for headways. Garwood derived an exact distribution for the delay. The next discussion of the delay problem was given by Raff, [3], who also assumed a negative exponential gap distribution and a gap acceptance function given by a step function, i.e., cross if the gap is greater than a given $T_{c}$. The same problem was taken up in much greater detail at about the same time by Tanner [4] who derived a large number of results for the statistics of the size of the queue of pedestrians. He assumed that the pedestrians' arrival distribution was Poisson, as well as that of the traffic, and calculated such quantities as the expected number of pedestrians crossing between successive cars, the size of the queue at a random time, and others which will be reproduced in the present paper. The basic assumption made by Tanner is that of a step function distribution for the gap acceptance function both for an individual pedestrian and for the group. We might note that the process described by Tanner, and indeed that which is described below in the following manuscript, is a bulk service process in which a stationary state is always reached for all gap distributions observed in moderate traffic. A later contribution to the pedestrian delay problem was made by Mayne, [5], who showed, by fairly involved calculations, how to calculate the delay to a single pedestrian when the gap distribution was other than Poisson, but otherwise assuming a step gap acceptance function.

All of the work mentioned above was carried out by means of combinatorial arguments, which, except in the case of a negative exponential gap distribution, were quite complicated. Recent work on delay problems has proceeded from the observation that the passage of a car on the main highway is a regeneration point, and the techniques of renewal theory can be used to solve delay problems in a way which almost completely eliminates combinatorial reasoning from the solution. As an example of papers which make use of renewal-theoretic techniques we cite [6-9]. Because the solution of delay problems has now been freed of the complications of the earlier analyses it is

\footnotetext{
*Present address: Rockefeller Institute, New York, N.Y.

${ }^{1}$ Figures in brackets indicate the literature references at the end of this paper.
} 
natural to attack more realistic situations. Thus, in the first paper on renewal theory as applied to the delay problem, not only was it possible to relax the restriction of a negative exponential gap distribution (this had already been done by Mayne) but it was also possible to take into account a gap acceptance function which was not a step function. Specifically, one can define a probability $\alpha(t)$, such that an individual will cross the highway with probability $\alpha(t)$ when the gap which confronts him is of duration $t$. Actual measurements of $\alpha(t)$, both on pedestrian and vehicular traffic, indicate that $\alpha(t)$ is a continuous function of $t$ rather than a step function, [10-12]. A typical form found in measurements on the vehicular gap acceptance probability is

$$
\begin{aligned}
\alpha(t) & =0 \quad 0 \leqslant t \leqslant T_{c} \\
& =1-\exp \left[-\beta\left(t-T_{c}\right)\right] \quad t \geqslant T_{c} .
\end{aligned}
$$

The theory which has been developed and partially checked against experiment applies to a single driver at a stop sign confronted by a single lane of homogeneous traffic. Very little is known about driver behavior when confronted by a multilane highway, although it is not difficult to extend the theory to the case where the waiting driver considers the situation in each lane independently. One can also solve for the moments of the delay distribution for a simple model of nonhomogeneous traffic in which there are $N$ types of cars and the gap distribution between successive cars depends on the types involved, [6].

While the single car, or single pedestrian delay distribution is a handy function for the traffic engineer to know, it has little bearing on the queueing properties of the system. This is a difficult problem to deal with when the queues are considered to be those of cars, who must necessarily form into a line, and may be released in groups whose size depends on the size of the gap in traffic on the main highway. In this situation, where it is assumed that the second car in line takes a nonnegligible time to reach the head of the line when the first car has crossed, the traffic on the main highway may indeed be so heavy as to cause an unlimited buildup of a queue on the feeder road. In the pedestrian queue first studied by Tanner all of the pedestrians in the queue see the gap simultaneously and can cross simultaneously. It is this feature that insures that except for pathologic and obviously unrealistic gap distributions, the queue will have steady state properties.

It is the purpose of this paper to reconsider the pedestrian queueing problem, eliminating the restriction to a negative exponential gap distribution and using a gap acceptance probability which is not necessarily a step function. It will be seen that by using the techniques associated with imbedded Markov chains (first introduced into this problem area by Oliver and Bisbee, [7]), the resulting calculations become simple, because of the particular form found for the transition matrix, when it is assumed that the gap acceptance function for a group of pedestrians is the same as that for a single pedestrian. We shall calculate the probability generating functions and the first two moments for the number of pedestrians in queue at each regeneration point, the number in queue at a random time, and the number crossing after a random vehicle.

\section{General Theory}

We begin by considering the following specification of the situation to be studied. We assume:

1. Poisson arrival of pedestrians, with arrival rate $\lambda$.

2. General stationary headway distribution in which successive gaps are independent random variables described by a density $\varphi(t)$.

3. The gap acceptance function for each pedestrian is $\alpha(t)$.

The first and simplest problem for pedestrian queueing is the study of the imbedded Markov chain, in which we take as the regeneration points for the process, the times of departure of cars on the highway from a point directly in front of the group of pedestrians. We shall calculate the elements of the transition matrix $\mathbf{P}=\left(p_{n m}\right)$ in several situations. The element $p_{n m}$ represents the 
probability that if there are $n$ pedestrians in queue at one regeneration point, there will be $m$ pedestrians in queue at the succeeding regeneration point. To calculate $p_{n m}$ we must be slightly more precise about our definition of regeneration points. It will be assumed that if a car passes in front of the queue at time $t$, any pedestrians who choose to cross will do so at a time $t+0$.

The problem which corresponds to those treated by previous authors is that in which either a pedestrian crosses on arrival at the intersection (i.e., he is not delayed at all) or else the whole group of pedestrians in queue decides to cross in a body when a new gap presents itself. The gap acceptance function for the group is taken to be that for the individual. We shall now calculate the $p_{n m}$ for this situation.

There are two cases to be examined. When $n$ is greater than $m$ the transition $n \rightarrow m$ can take place only as $n \rightarrow 0 \rightarrow m$, that is, first the queue empties entirely and then $m$ or more pedestrians arrive of which $m$ choose to join the queue. When $m$ is greater than or equal to $n$ the transition $n \rightarrow m$ can take place in two ways. The first is $n \rightarrow 0 \rightarrow m$ as already discussed, and the second is $n \rightarrow m$. In this second possibility the pedestrians in queue choose not to cross the road as the car passes and $m-n$ pedestrians arrive who choose to join the queue.

Since we are assuming Poisson arrival of pedestrians, the arrival times can be considered to be uniformly distributed in the interval $(0, t)$ for any $t,[14]$. Thus, the probability that a pedestrian will see an initial gap of $(x, x+d x)$ units of time will be $d x / t$. Therefore, the probability of immediate crossing by an arbitrary arrival in this interval is $\alpha^{*}(t)$, where

$$
\alpha^{*}(t)=\frac{1}{t} \int_{0}^{t} \alpha(x) d x
$$

$\alpha^{*}(t)$ is the average of $\alpha(t)$ taken with a uniform weight over the interval. The matrix element $p_{n m}$ for $n>m$ is therefore

$$
\begin{aligned}
p_{n m}^{(1)} & =\sum_{k=0}^{\infty} \int_{0}^{\infty} \alpha(t) \frac{(\lambda t)^{k+m}}{(k+m) !} e^{-\lambda t}\left(\begin{array}{c}
k+m \\
m
\end{array}\right)\left[\alpha^{*}(t)\right]^{k}\left[1-\alpha^{*}(t)\right]^{m} \varphi(t) d t \\
& =\int_{0}^{\infty} \alpha(t) e^{-\lambda t[1-\alpha *(t)]} \frac{\left[\lambda t\left(1-\alpha^{*}(t)\right)\right]^{m}}{m !} \varphi(t) d t, n>m .
\end{aligned}
$$

The derivation of this relation is as follows: The transition $n \rightarrow 0$ (the entire queue crosses with the passage of a car) takes place with probability $\alpha(t) ; k+m$ pedestrians arrive in the gap of $t$ units, with probability $e^{-\lambda t}(\lambda t)^{k+m} /(k+m)$ !; of these $m$ remain in queue, where $m$ is a random variable with a binomial distribution with probability $1-\alpha^{*}(t)$. Notice that the second line can be summarized by assuming a Poisson arrival rate on a reduced time scale defined by

$$
T(t)=t\left[1-\alpha^{*}(t)\right]=\int_{0}^{t}(1-\alpha(u)) d u .
$$

When $m$ is greater than or equal to $n$, another term is needed in addition to that given in eq (2.2). This term is needed to cover the case of the direct transition $n \rightarrow m$ and is

$$
\begin{aligned}
p_{n m}^{(2)} & =\sum_{k=0}^{\infty} \int_{0}^{\infty}[1-\alpha(t)] e^{-\lambda t} \frac{(\lambda t)^{k+m-n}}{(k+m-n) !}\left(\begin{array}{c}
k+m-n \\
m-n
\end{array}\right)\left[\alpha^{*}(t)\right]^{k+m-n}\left[1-\alpha^{*}(t)\right]^{m-n} \varphi(t) d t \\
& =\int_{0}^{\infty}[1-\alpha(t)] \frac{(\lambda T(t))^{m-n}}{(m-n) !} e^{-\lambda T(t)} \varphi(t) d t
\end{aligned}
$$


where $T(t)$ is defined in eq (2.3). The transition matrix $\mathbf{P}$ is therefore

$$
\mathbf{P}=\left[\begin{array}{ccccc}
\Delta_{0}+\epsilon_{0} & \Delta_{1}+\epsilon_{1} & \Delta_{2}+\epsilon_{2} & \Delta_{3}+\epsilon_{3} & \ldots \\
\Delta_{0} & \Delta_{1}+\epsilon_{0} & \Delta_{2}+\epsilon_{1} & \Delta_{3}+\epsilon_{2} & \ldots \\
\Delta_{0} & \Delta_{1} & \Delta_{2}+\epsilon_{0} & \Delta_{3}+\epsilon_{1} & \ldots \\
\Delta_{0} & \Delta_{1} & \Delta_{2} & \Delta_{3}+\epsilon_{0} & \ldots \\
. & . & . & . & \\
. & . & . & . & \\
. & . & . & . &
\end{array}\right]
$$

where $^{2}$

$$
\begin{aligned}
\Delta_{k} & =\int_{0}^{\infty} \alpha(t) \varphi(t) \frac{(\lambda T)^{k}}{k !} e^{-\lambda T} d t \\
\epsilon_{k} & =\int_{0}^{\infty}[1-\alpha(t)] \varphi(t) \frac{(\lambda T)^{k}}{k !} e^{-\lambda T} d t .
\end{aligned}
$$

The gross properties of this Markov chain are easily deduced. Let us denote by $\boldsymbol{\theta}(n)$ the vector of state probabilities after the passage of the $n$th car, where

$$
\theta(n)=\left(\theta_{0}(n), \theta_{1}(n), \ldots\right)
$$

The vector $\theta(n)$ satisfies

$$
\boldsymbol{\theta}(n+1)=\boldsymbol{\theta}(n) \mathbf{P}
$$

or

$$
\theta(n)=\theta(0) \mathbf{P}^{n}
$$

Because of the simple form of $\mathbf{P}$ shown in eq (2.5), eq (2.8) is most conveniently written in component form

$$
\theta_{k}(n+1)=\Delta_{k}+\sum_{j=0}^{k} \theta_{j}(n) \epsilon_{k-j}
$$

which suggests the use of generating functions. Defining the generating functions

$$
\begin{gathered}
\Psi_{n}(s)=\sum_{k=0}^{\infty} \theta_{k}(n) s^{k}, \quad \epsilon(s)=\sum_{k=0}^{\infty} \epsilon_{k} s^{k} \\
\Delta(s)=\sum_{k=0}^{\infty} \Delta_{k} s^{k},
\end{gathered}
$$

we find

and by iteration that

$$
\Psi_{n+1}(s)=\Delta(s)+\epsilon(s) \Psi_{n}(s)
$$

$$
\Psi_{n}(s)=\epsilon^{n}(s) \Psi_{0}(s)+\Delta(s) \frac{1-\epsilon^{n}(s)}{1-\epsilon(s)} .
$$

It is readily verified that

$$
\begin{aligned}
& \Delta(s)=\int_{0}^{\infty} \alpha(t) \varphi(t) e^{-\lambda T(t)(1-s)} d t \\
& \epsilon(s)=\int_{0}^{\infty}[1-\alpha(t)] \varphi(t) e^{-\lambda T(t)(1-s)} d t .
\end{aligned}
$$

${ }^{2}$ Although the derivation of $p_{0 j}=\Delta_{j}+\epsilon_{j}$ is not quite correct, since one cannot speak of a group of 0 persons choosing to cross the road, the result is nonetheless correct, since $\Delta_{j}+\epsilon_{j}$ is independent of $\alpha(t)$. 
It would be difficult to solve explicitly for all of the components of $\theta(n)$. However, it is not difficult to find an expression for the moments. Let us denote by $\mu_{k}(n)$ the $k$ th ordinary moment, that is

$$
\mu_{k}(n)=\sum_{m=0}^{\infty} m^{k} \theta_{m}(n)
$$

Noting that

we find for the first two moments

$$
\epsilon(1)+\Delta(1)=1
$$

$$
\begin{aligned}
\mu_{1}(n)=\mu_{1}(\infty)+\epsilon^{n}(1)\left[\mu_{1}(0)\right. & \left.-\mu_{1}(\infty)\right] \\
\mu_{2}(n) & =\mu_{1}(n)+\epsilon^{n}(1)\left[\mu_{2}(0)-\mu_{1}(0)\right]+\frac{1-\epsilon^{n}(1)}{1-\epsilon(1)} \int_{0}^{\infty}(\lambda T)^{2} \varphi(t) d t \\
+ & 2\left[\frac{1-\epsilon^{n}(1)}{1-\epsilon(1)} \mu_{1}(\infty)+n \epsilon^{n-1}(1)\left(\mu_{1}(0)-\mu_{1}(\infty)\right)\right] \int_{0}^{\infty} \lambda T(1-\alpha(t)) \varphi(t) d t
\end{aligned}
$$

where

and

$$
\epsilon(1)=\int_{0}^{\infty}[1-\alpha(t)] \varphi(t) d t
$$

$$
\mu_{1}(\infty)=\frac{\int_{0}^{\infty} \lambda T(t) \varphi(t) d t}{1-\epsilon(1)}=\frac{\int_{0}^{\infty} \lambda T(t) \varphi(t) d t}{\int_{0}^{\infty} \alpha(t) \varphi(t) d t} .
$$

The equilibrium value of the second moment, $\mu_{2}(\infty)$ is given by

$$
\mu_{2}(\infty)=\mu_{1}(\infty)+\frac{\int_{0}^{\infty}(\lambda T)^{2} \varphi(t) d t}{1-\epsilon(1)}+\frac{2}{(1-\epsilon(1))^{2}}\left\{\int_{0}^{\infty} \lambda T[1-\alpha(t)] \varphi(t) d t\right\}\left\{\int_{0}^{\infty} \lambda T \varphi(t) d t\right\} .
$$

By these methods we can determine the distribution of the several parameters discussed by Tanner and by Mayne. The probability that there are $n$ pedestrians in queue after a random vehicle is $\theta_{n}(\infty)$. Hence the expected number of pedestrians crossing after a random vehicle passes is

$$
\sum_{n=0}^{\infty} n \theta_{n}(\infty) \int_{0}^{\infty} \alpha(t) \varphi(t) d t=\mu_{1}(\infty) \int_{0}^{\infty} \alpha(t) \varphi(t) d t
$$

The $k$ th moment of the distribution is similarly equal to $\mu_{k}(\infty) \int_{0}^{\infty} \alpha(t) \varphi(t) d t$.

The probability that there are no pedestrians in queue when the $n$th vehicle passes is

where

$$
\theta_{0}(n)=\Psi_{n}(0)=\epsilon^{n}(0) \Psi_{0}(0)+\Delta(0) \frac{1-\epsilon^{n}(0)}{1-\epsilon(0)}
$$

$$
\begin{aligned}
& \epsilon(0)=\epsilon_{0}=\int_{0}^{\infty} \alpha(t) \varphi(t) e^{-\lambda T(t)} d t \\
& \Delta(0)=\Delta_{0}=\int_{0}^{\infty}[1-\alpha(t)] \varphi(t) e^{-\lambda T(t)} d t .
\end{aligned}
$$

At equilibrium $(n=\infty)$ we have the result

$$
\theta_{0}(\infty)=\frac{\Delta(0)}{1-\epsilon(0)}=\frac{\Delta_{0}}{1-\epsilon_{0}} .
$$


The probability density for the amount of time that any single pedestrian is delayed will be denoted by $\Omega(t)$, and can be immediately taken from the results of reference [6], since each pedestrian can be regarded as not being influenced by the presence of any others. Let us denote the Laplace transform of any function, say $F(t)$, by the same function with argument $s$ and an asterisk, e.g.,

$$
F^{*}(s)=\int_{0}^{\infty} e^{-s t} F(t) d t .
$$

Then it is shown in [6] that

$$
\Omega^{*}(s)=\int_{0}^{\infty} \alpha(t) \varphi_{0}(t) d t+\frac{\zeta_{0}^{*}(s)}{1-\zeta^{*}(s)} \int_{0}^{\infty} \alpha(t) \varphi(t) d t
$$

where

$$
\begin{gathered}
\zeta(t)=\varphi(t)[1-\alpha(t)] \\
\zeta_{0}(t)=\varphi_{0}(t)[1-\alpha(t)]
\end{gathered}
$$

and where $\varphi_{0}(t)$ is the density of the first gap given by

$$
\varphi_{0}(t)=\int_{t}^{\infty} \varphi(x) d x / \int_{0}^{\infty} x \varphi(x) d x .
$$

Expressions for the first two moments of the delay density are given in [6] and are repeated here for reference:

$$
\begin{aligned}
& \bar{t}=\eta_{01}+\frac{\eta_{00}}{1-\eta_{10}} \eta_{11} \\
& \overline{t^{2}}=\eta_{02}+\frac{2 \eta_{01} \eta_{11}+\eta_{00} \eta_{12}}{1-\eta_{10}}+\frac{2 \eta_{00} \eta^{2}{ }_{11}}{\left(1-\eta_{10}\right)^{2}}
\end{aligned}
$$

where

$$
\begin{aligned}
& \eta_{0 j}=\int_{0}^{\infty}{ }^{j} \zeta_{0}(t) d t=\int_{0}^{\infty} t^{j} \varphi_{0}(t)[1-\alpha(t)] d t \\
& \eta_{1 j}=\int_{0}^{\infty} t^{j} \zeta(t) d t=\int_{0}^{\infty} t^{j} \varphi(t)[1-\alpha(t)] d t .
\end{aligned}
$$

Another quantity of interest that can be derived from the present analysis is the distribution of the number of pedestrians crossing between two consecutive vehicles. Let $\pi_{k}$ be the probability that $k$ pedestrians cross between two successive vehicles. In solving for $\pi_{k}$ we must take two cases into account; either the group of pedestrians initially in queue (when the first car passes the group) cross together, or it does not. The probability that $k$ or more pedestrians arrive in a time $t$ (the time delay between the arrival between two successive cars) and that exactly $k$ of these cross, is

$$
\sum_{m=0}^{\infty} e^{-\lambda t} \frac{(\lambda t)^{k+m}}{(k+m) !}\left(\begin{array}{c}
k+m \\
m
\end{array}\right)\left[\alpha^{*}(t)\right]^{k}\left[1-\alpha^{*}(t)\right]^{m}=\frac{\left[\lambda t \alpha^{*}(t)\right]^{k}}{k !} e^{-\lambda t \alpha^{*}(t)}=e^{-\lambda(t-T(t))} \frac{[\lambda(t-T(t))]^{k}}{k !}
$$

where $T(t)$ is defined in eq (2.3). Hence, considering separately the two cases mentioned above, we have, for the probability that $k$ pedestrians cross assuming that the group initially in queue crosses.

$$
\pi_{k}^{(1)}=\sum_{j=0}^{k} \theta_{j}(\infty) \int_{0}^{\infty} \alpha(t) \varphi(t) \frac{\left[\lambda t \alpha^{*}(t)\right]^{k-j}}{(k-j) !} e^{-\lambda t \alpha^{*}(t)} d t
$$

In the second case, where it is assumed that the group in queue does not cross we have,

$$
\pi_{k}^{(2)}=\int_{0}^{\infty}[1-\alpha(t)] \varphi(t) \frac{\left[\lambda t \alpha^{*}(t)\right]^{k}}{k !} e^{-\lambda t \alpha^{*}(t)} d t
$$


In this situation the size of the initial queue is obviously irrelevant. Finally $\pi_{k}$ is just the sum

$$
\pi_{k}=\pi_{k}^{(1)}+\pi_{k}^{(2)}
$$

The moments can be derived from the generating function

$$
\pi(s)=\sum_{k=0}^{\infty} \pi_{k} s^{k}=\int_{0}^{\infty} \alpha(t) \varphi(t) \psi_{\infty}(s) e^{-\lambda t \alpha^{*}(t)(1-s)} d t+\int_{0}^{\infty}[1-\alpha(t)] \varphi(t) e^{-\lambda t \alpha^{*}(t)(1-s)} d t .
$$

The first two moments of this distribution, denoted by $\overline{n_{1}}$ and $\overline{n_{2}}$ are

$$
\begin{aligned}
\overline{n_{1}}=\mu_{1}(\infty) \int_{0}^{\infty} \alpha(t) \varphi(t) d t+ & \int_{0}^{\infty} \lambda t \alpha^{*}(t) \varphi(t) d t=\lambda \int_{0}^{\infty} t \varphi(t) d t \\
& \overline{n_{2}}=\mu_{2}(\infty) \int_{0}^{\infty} \alpha(t) \varphi(t) d t+2 \mu_{1}(\infty) \int_{0}^{\infty} \lambda t \alpha^{*}(t) \alpha(t) \varphi(t) d t \\
& +\int_{0}^{\infty}\left[\lambda t \alpha^{*}(t)\right]^{2} \varphi(t) d t+\overline{n_{1}} .
\end{aligned}
$$

Similar calculations enable us to find the probability that there are $m$ pedestrians in queue at a random time, not necessarily just after the passage of a car. As before we shall assume that the event "passage of a car" is a regeneration point for the process, and calculate a set of transition probabilities, $\left\{\lambda_{n m}\right\}$, where $\lambda_{n m}$ is the probability that there are $m$ pedestrians in queue at a random time, given that there were $n$ in queue at the last regeneration point. For the present calculation we assume that the gap in which the random time finds itself is of duration $t$, and that the last regeneration point occurred $y$ units of time ago, where clearly $y \leqslant t$.

We first calculate the probability density for the duration of the gap, $t$, in which the instant find: itself. Let $w(x) d x$ be the probability for the occurrence of a regeneration point to occur in $(x, x+d x)$. Let $\rho(t, y) d t$ be the probability that a point at $y$ is covered by a gap whose duration satisfies $t \leqslant G<t+d t$. We shall only be interested in the limit $y \rightarrow \infty$. The probability that any gap has duration $G$ such that $t \leqslant G<t+d t$ is just $\varphi(t) d t$. However, we must account for the fact that the last regeneration point lies in the interval ${ }^{3} y, y-t$. This probability is just $\int_{y-t}^{y} w(x) d x$, hence the probability density for the size of gap including a random time point is

$$
\rho(t, y)=\varphi(t)\left[\int_{0}^{y} w(x) d x-\int_{0}^{y-t} w(x) d x\right]+0(1)
$$

in the limit of $y \rightarrow \infty$. Under quite general conditions on $\varphi(t)$ which are always met in practice it can be shown by the methods of renewal theory, [13], that

$$
\int_{0}^{y} w(x) d x \sim \frac{y}{\nu_{1}}
$$

in the limit of $y \rightarrow \infty$, where

$$
\nu_{1}=\int_{0}^{\infty} t \varphi(t) d t
$$

Hence we finally find

$$
\rho(t, \infty)=\frac{t \varphi(t)}{\nu_{1}}
$$

This result has been derived in a slightly different manner by Oliver and Jewell, [15].

\footnotetext{
${ }^{3}$ We tacitly assume that $y$ is chosen so large that $y>t$. A more complete result which does not use this assumption is easy to prove, but in what follows we are only interested in the equilibrium result.
} 
We now define a function $\delta_{n m}(y, t)$ by $\delta_{n m}(y, t) d y=$ probability that there were $n$ pedestrians in queue at the last regeneration point which occurred in $(t-y, t-y+d y)$, and that $m$ pedestrians are presently in queue. The quantities which we seek, the $\lambda_{n m}$ are related to $\delta_{n m}(y, t)$ by

$$
\lambda_{n m}=\int_{0}^{\infty} \rho(t) d t \int_{0}^{t} \frac{d y}{t} \delta_{n m}(y, t)
$$

since the time of observation is uniformly distributed over the gap duration. An expression for $\delta_{n m}(y, t)$ can immediately be written down by exactly following the argument which led to eq (2.5). The final result is

$$
\begin{aligned}
\delta_{n m}(y, t)= & \alpha(t) e^{-\lambda T^{+}(y, t)} \frac{\left[\lambda T^{+}(y, t)\right]^{n}}{n !} n>m \\
\delta_{n m}(y, t)= & \alpha(t) e^{-\lambda T^{+}(y, t)} \frac{\left[\lambda T^{+}(y, t)\right]^{n}}{n !} m \geqslant n \\
& +(1-\alpha(t)) e^{-\lambda T^{+}(y, t)} \frac{\left[\lambda T^{+}(y, t)\right]^{m-n}}{(m-n) !}
\end{aligned}
$$

where

$$
\lambda T^{+}(y, t)=\lambda \int_{t-y}^{t}(1-\alpha(u)) d u .
$$

Hence, finally, $\lambda_{n m}$ can be expressed as the sum of two terms:

$$
\begin{aligned}
\lambda_{n m} & =A_{m} & & n>m \\
& =A_{m}+B_{m-n} & & m \geqslant n
\end{aligned}
$$

where, combining eqs (2.38), (2.40), and (2.42)

$$
\begin{aligned}
& A_{n}=\frac{1}{n ! \nu_{1}} \int_{0}^{\infty} \alpha(t) \varphi(t) d t \int_{0}^{t}\left[\lambda T^{+}(y, t)\right]^{n} e^{-\lambda T^{+}(y, t)} d y \\
& B_{r}=\frac{1}{r ! \nu_{1}} \int_{0}^{\infty}(1-\alpha(t)) \varphi(t) d t \int_{0}^{t}\left[\lambda T^{+}(y, t)\right]^{r} e^{-\lambda T^{+}(y, t)} d y .
\end{aligned}
$$

Let us denote by $c_{m}$ the probability that there are $m$ pedestrians in queue at a random time. This probability is expressible in terms of the $\theta_{n}(\infty)$ as

$$
c_{m}=\sum_{n=0}^{\infty} \theta_{n}(\infty) \lambda_{n m}=A_{m}+\sum_{n=0}^{m} \theta_{n}(\infty) B_{m-n} .
$$

Since this equation is in convolution form we can profitably write down the generating function for $c_{m}$, call it $C(s)$, and relate it to the generating functions of $A_{m}$ and $B_{m}, A(s)$ and $B(s)$ respectively. In this way we are led to

$$
C(s)=A(s)+\frac{\Delta(s)}{1-\epsilon(s)} B(s)
$$

where we have used eq (2.13) for the generating function of the $\theta_{n}(\infty)$. The generating functions $A(s)$ and $B(s)$ are explicitly

$$
\begin{aligned}
& A(s)=\frac{1}{\nu_{1}} \int_{0}^{\infty} \alpha(t) \varphi(t) d t \int_{0}^{t} e^{-\lambda T^{+}(y, t)(1-s)} d y \\
& B(s)=\frac{1}{\nu_{1}} \int_{0}^{\infty}(1-\alpha(t)) \varphi(t) d t \int_{0}^{t} e^{-\lambda T^{+}(y, t)(1-s)} d y .
\end{aligned}
$$


For the first two moments we find by differentiation

$$
\begin{aligned}
\bar{m}=\frac{\lambda}{\nu_{1}} \int_{0}^{\infty} \varphi(t) d t \int_{0}^{t} T^{+}(y, t) d y+\frac{\mu_{1}(\infty)}{\nu_{1}} \int_{0}^{\infty} t(1-\alpha(t)) \varphi(t) d t \\
\bar{m}^{2}=\bar{m}+\frac{\lambda^{2}}{\nu_{1}} \int_{0}^{\infty} \varphi(t) d t \int_{0}^{t}\left[T^{+}(y, t)\right]^{2} d y \\
+\frac{2 \mu_{1}(\infty) \lambda}{\nu_{1}} \int_{0}^{\infty}(1-\alpha(t)) \varphi(t) d t \int_{0}^{t} T^{+}(y, t) d y \\
+\frac{\mu_{2}(\infty)}{\nu_{1}} \int_{0}^{\infty} t(1-\alpha(t)) \varphi(t) d t .
\end{aligned}
$$

When $\alpha(t)$ is a step function,

$$
\begin{aligned}
\alpha(t) & =0 & & t<T_{c} \\
& =1 & & t>T_{c}
\end{aligned}
$$

the double integrals can be reduced to single integrals by introducing the explicit form for $T^{+}(y, t)$ :

$$
\begin{aligned}
& T^{+}(y, t)=0 \quad t>T_{c}, \quad t-y>T_{c} \\
& =T_{c}-t+y \quad t>T_{c}, \quad t-y<T_{c} \\
& =y \quad t<T_{c} \text {. }
\end{aligned}
$$

When this form for $T^{+}(y, t)$ is substituted into eq (2.47), we obtain the results

$$
\begin{aligned}
\bar{m}=\frac{1}{2 \nu_{1}} \int_{0}^{T_{c}} t^{2} \varphi(t) d t+\frac{\lambda T_{c}^{2}}{2 \nu_{1}} \Phi\left(T_{c}\right)+\frac{\mu_{1}(\infty)}{\nu_{1}} \int_{0}^{T_{c}} t \varphi(t) d t \\
\bar{m}^{2}=\bar{m}+\frac{\lambda^{2}}{3 \nu_{1}} \int_{0}^{T_{c}} t^{3} \varphi(t) d t+\frac{\lambda^{2} T_{c}^{3}}{3 \nu_{1}} \Phi\left(T_{c}\right) \\
+\frac{\mu_{1}(\infty) \lambda}{\nu_{1}} \int_{0}^{T_{c}} t^{2} \varphi(t) d t \\
+\frac{\mu_{2}(\infty)}{\nu_{1}} \int^{T_{c}} t \varphi(t) d t
\end{aligned}
$$

where

$$
\Phi(t)=\int_{t}^{\infty} \varphi(x) d x
$$

\section{Some Applications}

We shall now apply some of the preceding general results to the specific distributions

$$
\begin{aligned}
\varphi_{m}(t) & =\sigma e^{-\sigma t} \frac{(\sigma t)^{m}}{m !} & & m=0,1,2, \ldots \\
\alpha(t) & =0 & & t<T_{c} \\
& =1-e^{-\beta\left(t-T_{c}\right)} & & t \geqslant T_{c} .
\end{aligned}
$$

When $\beta$ tends to infinity this form of $\alpha(t)$ tends to the step function

$$
\begin{aligned}
\alpha(t) & =0 & & t<T_{c} \\
& =1 & & t \geqslant T_{c}
\end{aligned}
$$


which has been used extensively in previous treatments of this subject. It is easily verified that for this choice of $\alpha(t)$ we have for $\alpha^{*}(t)$ :

and

$$
\begin{array}{rlrl}
\alpha^{*}(t) & = & 0 \quad t<T_{c} \\
& =\frac{1}{\beta t}\left[\beta t-\beta T_{c}-1+e^{-\beta\left(t-T_{c}\right)}\right] \quad t>T_{c}
\end{array}
$$

$$
\begin{aligned}
T(t) & =t\left[1-\alpha^{*}(t)\right] \\
& =t \quad t<T_{c} \\
& =\frac{1}{\beta}\left[1+\beta T_{c}-e^{-\beta\left(t-T_{c}\right)}\right] \quad t \geqslant T_{c}
\end{aligned}
$$

A quantity which frequently occurs in the calculations is $\epsilon(1)$, which for the present set of models is

$$
\begin{aligned}
\boldsymbol{\epsilon}(1) & =\int_{0}^{\infty} \varphi_{m}(t)[1-\alpha(t)] d t \\
& =\sigma \int_{0}^{T_{c}} e^{-\sigma t} \frac{(\sigma t)^{m}}{m !} d t+\sigma \int_{T_{c}}^{\infty} e^{-\sigma t} \frac{(\sigma t)^{n}}{n !} e^{-\beta\left(t-T_{c}\right)} d t \\
& =1-G_{m}\left(\sigma T_{c}\right)+\left(\frac{\sigma}{\sigma+\beta}\right)^{m+1} e^{\beta T_{c}} G_{m}\left[(\sigma+\beta) T_{c}\right]
\end{aligned}
$$

where

$$
G_{m}(x)=e^{-x}\left(1+x+\frac{x^{2}}{2 !}+\ldots+\frac{x^{m}}{m !}\right)
$$

When $\beta=\infty$, the expression for $\epsilon(1)$ becomes

$$
\epsilon(1)=1-G_{m}\left(\sigma T_{c}\right)
$$

An expression for the quantity

$$
\int_{0}^{\infty} \lambda T(t) \varphi_{m}(t) d t
$$

which appears in the formula for $\mu_{1}(\infty)$ (of eq (2.19)) is

$$
\begin{aligned}
\int_{0}^{\infty} \lambda T(t) \varphi_{m}(t) d t=(m+1) \frac{\lambda}{\sigma}\left[1-G_{m+1}\right. & \left.\left(\sigma T_{c}\right)\right] \\
& +\frac{\lambda}{\beta}\left(1+\beta T_{c}\right) G_{m}\left(\sigma T_{c}\right)-\frac{\lambda}{\beta} e^{\beta T_{c}}\left(\frac{\sigma}{\sigma+\beta}\right)^{m+1} G_{m}\left[(\sigma+\beta) T_{c}\right] .
\end{aligned}
$$

When $\beta=\infty$ this expression reduces to

$$
\int_{0}^{\infty} \lambda T(t) \varphi_{m}(t) d t=(m+1) \frac{\lambda}{\sigma}\left[1-G_{m+1}\left(\sigma T_{c}\right)\right]+\lambda T_{c} G_{m}\left(\sigma T_{c}\right)
$$

As a specific example of the use of these formulas, let us specialize to the case $m=0$, i.e.,

$$
\varphi(t)=\sigma e^{-\sigma t}
$$

where $\sigma$ is the expected number of cars passing per unit time. Then it is easily found that

$$
\epsilon(1)=1-\frac{\beta}{\sigma+\beta} e^{-\sigma T_{c}}
$$

and

$$
\mu_{1}(\infty)=\lambda\left(\frac{1}{\sigma}+\frac{1}{\beta}\right)\left(e^{\sigma T} c-\frac{\beta}{\beta+\sigma}\right)
$$




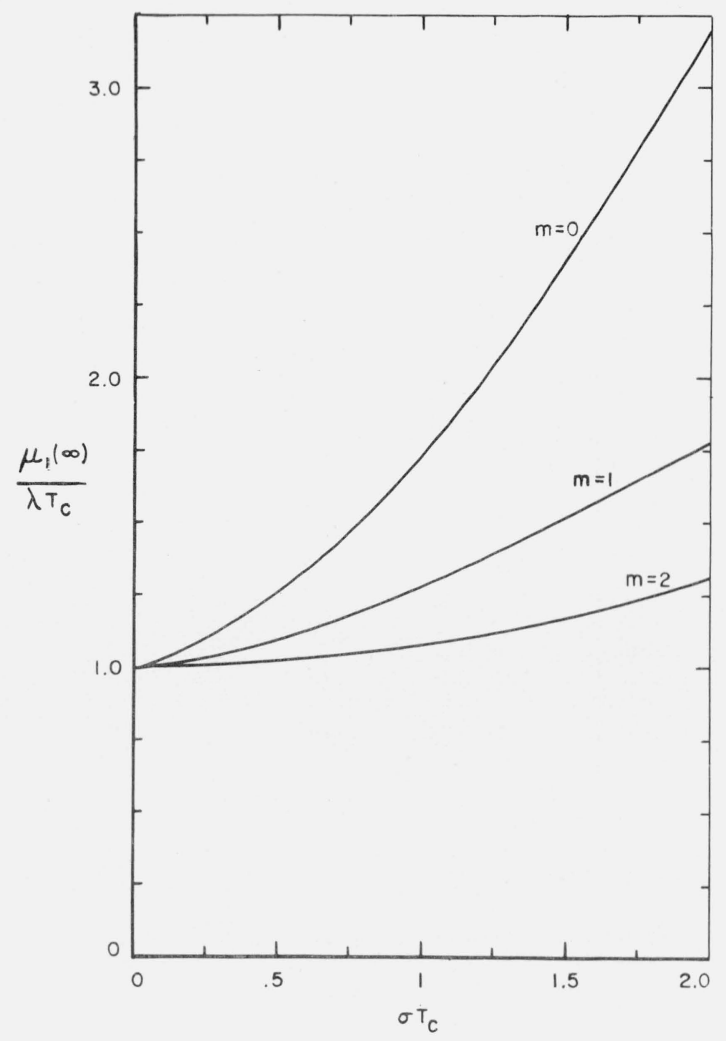

FIGURE 1. Plot of normalized expected queue length as a function of normalized traffic intensity.

is the equilibrium value of the expected number of people in queue at a regeneration point. When $\beta=\infty$ we obtain the value given by Tanner for the case of a negative exponential headway distribution:

$$
\mu_{1}(\infty)=\frac{\lambda}{\sigma}\left(e^{\sigma T_{c}}-1\right)
$$

We find in general for $\beta=\infty$, the value of $\mu_{1}(\infty)$ is

$$
\mu_{1}(\infty)=\lambda T_{c}+(m+1) \frac{\lambda}{\sigma}\left[\frac{1-G_{m+1}\left(\sigma T_{c}\right)}{G_{m}\left(\sigma T_{c}\right)}\right] .
$$

Several curves showing $\mu_{1}(\infty) /\left(\lambda T_{c}\right)$, the normalized expected number of pedestrians in queue at the passage of a random car are shown in figure 1. As $\sigma \rightarrow \infty$, i.e., the mean gap between vehicles decreases to zero, we find

$$
\mu_{1}(\infty)=\lambda T_{c}\left[\frac{(m+1) !}{\left(\sigma T_{c}\right)^{m+1}} e^{\sigma T_{c}}+0(1)\right] .
$$

When $\sigma \rightarrow 0$ we find

$$
\mu_{1}(\infty) \sim \lambda T_{c}\left[1+\frac{\left(\sigma T_{c}\right)^{m+1}}{m !(m+2)}\right] .
$$

The equilibrium probability that there are no pedestrians in queue when a random car passes is given in eq (2.24). When $\varphi(t)$ is given by $\varphi_{m}(t)$ of eq (3.1) and $\alpha(t)$ is a step function $\theta_{0}(\infty)$ is 


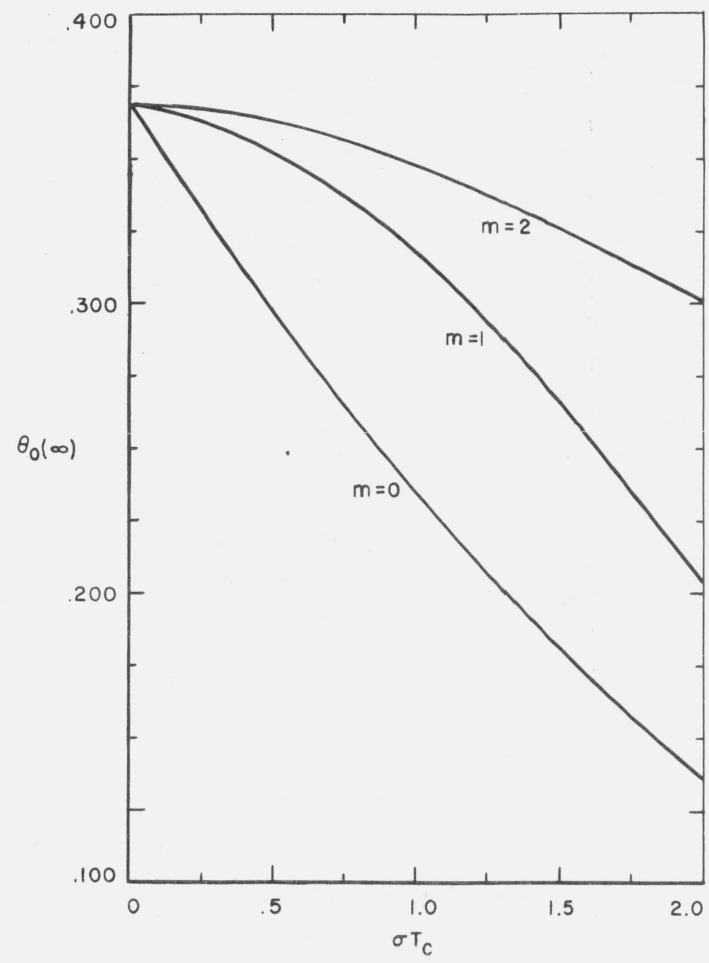

FigURE 2. Plot of probability of an empty queue as a function of normalized traffic intensity.

given by

$$
\theta_{0}(\infty)=\frac{e^{-\lambda T_{c}} G_{m}\left(\sigma T_{c}\right)}{1-\left(\frac{\sigma}{\sigma+\lambda}\right)^{m+1}\left(1-G_{m}\left\{(\sigma+\lambda) T_{c}\right\}\right)}
$$

In particular when $m=0$ this expression reduces to that derived by Tanner, [4],

$$
\theta_{0}(\infty)=\frac{\sigma+\lambda}{\lambda e^{(\sigma+\lambda) T} c+\sigma} .
$$

Figure 2 contains plots of $\theta_{0}(\infty)$ for $\lambda T_{c}=1$ and different values of $m$. When $\sigma \rightarrow \infty$ eq (3.17) yields the approximate result

$$
\theta_{0}(\infty)=\frac{\left(\sigma T_{c}\right)^{m}}{m !} \frac{e^{-(\lambda+\sigma) T_{c}}}{1-\left(\frac{\sigma}{\sigma+\lambda}\right)^{m+1}}+0\left[\left(\sigma T_{c}\right)^{m-1}\right]
$$

and when $\sigma \rightarrow 0$, or equivalently, traffic is light, eq (3.17) reduces to

$$
\theta_{0}(\infty)=e^{-\lambda T_{c}}\left[1-\left(\sigma T_{c}\right)^{m+1}\left\{\frac{1}{(m+1) !}-\frac{1}{\left(\lambda T_{c}\right)^{m+1}}+\frac{G_{m}\left(\lambda T_{c}\right)}{\left(\lambda T_{c}\right)^{m+1}}\right\}\right]+0\left(\left(\sigma T_{c}\right)^{m+1}\right) .
$$

In the first approximation we see that the probability that there are no pedestrians in queue does not depend on the vehicular traffic, but only on the pedestrian arrival rate.

The expected number of pedestrians crossing between two cars can be calculated from eq (2.35) When $\varphi(t)$ is of the form of eq (3.1), then detailed calculation leads to the result

$$
\bar{n}_{1}=(m+1) \frac{\lambda}{\sigma} .
$$


The expected size of the queue at a random time is given in the first line of eq (2.47). When $\varphi_{k}(t)$ and $\alpha(t)$ are of the form given in eq (3.1) we find

$$
\begin{aligned}
\bar{m}=\frac{\lambda}{2} \frac{(k+2)}{\sigma}\left[1-G_{k+2}\left(\sigma T_{c}\right)\right]+\frac{\lambda \sigma T_{c}^{2}}{2(k+1)} & G_{k}\left(\sigma T_{c}\right) \\
& +\left[1-G_{k+1}\left(\sigma T_{c}\right)\right]\left[\lambda T_{c}+(k+1) \frac{\lambda}{\sigma} \frac{1-G_{k+1}\left(\sigma T_{c}\right)}{G_{k}\left(\sigma T_{c}\right)}\right] .
\end{aligned}
$$

When $\sigma \rightarrow \infty$ this expression becomes approximately

$$
\bar{m}=\frac{(k+1) ! \lambda e^{\sigma T} c}{\sigma\left(\sigma T_{c}\right)^{k}}+0(1)
$$

and for $\sigma \rightarrow 0$ the expression goes into

$$
\bar{m}=\lambda T_{c}\left[1+0\left(\sigma T_{c}\right)\right]
$$

For the Poisson distribution we set $k=0$ and obtain the exact result

as derived by Tanner.

$$
\bar{m}=\frac{\lambda}{\sigma}\left(e^{\sigma T_{c}}-1-\sigma T_{c}\right)
$$

\section{Comments and Generalizations}

One of the weakest points of the preceding analysis is the assumption that a group of pedestrians will have the same gap acceptance probability as a single pedestrian. If we assume that there are, instead, an ensemble of gap acceptance probabilities $\left\{\alpha_{n}(t)\right\}$ then the form of $\mathbf{P}$ is not the same as that shown in eq (2.5), and it is necessary to discuss the resulting Markov chain ab initio. In particular it is no longer possible to have as simple a relation between generating functions as that given in eq (2.12). The more complete form for $\mathbf{P}$ is easily shown to be

$$
\mathbf{P}=\left[\begin{array}{cccc}
\Delta_{00}+\epsilon_{00} & \Delta_{01}+\epsilon_{01} & \Delta_{02}+\epsilon_{02} & \ldots \\
\Delta_{10} & \Delta_{11}+\epsilon_{11} & \Delta_{12}+\epsilon_{12} & \ldots \\
\Delta_{20} & \Delta_{21} & \Delta_{22}+\epsilon_{22} & \ldots \\
. & . & . & . \\
. & . & . & . \\
. & . & . & .
\end{array}\right]
$$

where

$$
\begin{aligned}
\Delta_{i j} & =\int_{0}^{\infty} \alpha_{i}(t) e^{-\lambda t}\left[1-\alpha_{i}^{*}(t)\right] \frac{\left[\lambda t\left(1-\alpha_{i}^{*}(t)\right)\right]^{j}}{j !} \varphi(t) d t \\
\epsilon_{i j} & =\int_{0}^{\infty}\left(1-\alpha_{i}(t)\right) e^{-\lambda t}\left[1-\alpha_{i}^{*}(t)\right] \frac{\left[\lambda t\left(1-\alpha_{i}^{*}(t)\right]^{j-i}\right.}{(j-i) !} \varphi(t) d t .
\end{aligned}
$$

Attempts to solve the steady state equations corresponding to the transition matrix of eq (4.1) have been unsuccessful. It is no longer clear in this situation that the queue will reach a steady state, although this will evidently be the case when $\alpha_{1}(t) \leqslant \alpha_{2}(t) \leqslant \alpha_{3}(t) \leqslant$. . . since we have seen that a steady state is reached when all of the $\alpha(t)$ are equal, and the inequality provides for faster servicing of the arriving pedestrians. 
There is one case of differential gap acceptance probabilities that can be solved. Let us assume that

$$
\alpha_{1}(t)=\beta(t), \alpha_{2}(t)=\alpha_{3}(t)=\ldots .=\alpha(t)
$$

that is, that group behavior will differ from individual behavior, although the gap acceptance probability for a group of greater than one will not depend on the size of the group. We shall examine the steady state equations only, i.e., the solution to the equation $\theta(\infty)=\theta(\infty) \mathbf{P}$. When these equations are written out in component form (omitting the infinity in the argument) we find

$$
\theta_{n}=\Delta_{n}+\sum_{r=0}^{n} \theta_{r} \epsilon_{n-r}+\theta_{1} \gamma_{n}
$$

where $\Delta_{j}$ and $\epsilon_{j}$ are just the functions defined in eq (2.6), and

$$
\begin{gathered}
\gamma_{n}=\int_{0}^{\infty} \varphi(t) e^{-\lambda T}[\beta(t)-\alpha(t)]\left[\frac{(\lambda T)^{n}}{n !}-\frac{(\lambda T)^{n-1}}{(n-1) !}\right] d t \\
n \geqslant 1 \\
\gamma_{0}=\int_{0}^{\infty} \varphi(t) e^{-\lambda T}[\beta(t)-\alpha(t)] d t
\end{gathered}
$$

and

$$
T(t)=\int_{0}^{t}(1-\beta(u)) d u
$$

The equation satisfied by the generating function is

$$
\Psi(s)=\frac{\Delta(s)+\theta_{1} \gamma(s)}{1-\epsilon(s)}
$$

where $\Psi(s)=\sum_{n=0}^{\infty} \theta_{n} s^{n}$ and $\gamma(s)=\sum_{n=0}^{\infty} \gamma_{n} s^{n}$. The right side of this equation still contains the unknown value of $\theta_{1}$, but this can be eliminated by differentiating both sides and setting $s$ equal to zero. With this procedure we find

$$
\theta_{1}=\frac{\Delta_{1}\left(1-\epsilon_{0}\right)+\Delta_{0} \epsilon_{1}}{\left(1-\epsilon_{0}\right)^{2}-\gamma_{1}\left(1-\epsilon_{0}\right)-\gamma_{0} \epsilon_{1}}
$$

which can then be substituted back into eq (4.7) to give the full expression for the generating function.

When it is assumed that the first $r$ of the $\alpha_{j}(t)$ differ, but the remaining $\alpha_{j}(t)$ are all equal, then $r$ of the $\theta$ 's appear in the expression for the generating function. These can be found by inverting an $r x r$ matrix. We will not pursue this line of inquiry any further since no experimental data exists which is relevant to this matter.

Another shortcoming of the model, even in the somewhat more general formulation of the last paragraph, is the fact that either none or all of the pedestrians in queue are assumed to cross at the same time. One can formally remedy this deficiency by defining a matrix $\left(\alpha_{n r}(t)\right)$ such that $\alpha_{n r}(t)$ is the probability that $r$ out of $n$ pedestrians will accept a gap of duration $t$. However, detailed calculations show that the resultant transition matrix is such that the stationary problem cannot be solved by generating function methods, and so far the solution to the problem is unknown.

It is possible by our methods to treat the queueing problems with bunched arrivals, where the 
bunches arrive in accordance with a Poisson process, but the number in each bunch is a random variable with a known distribution. Again, detailed calculation for this situation is hardly informative without any supporting data.

I thank Alan Goldman for his careful reading of this manuscript and for several suggestions which have been incorporated into the final draft.

\section{References}

[1] W. F. Adams, Road traffic considered as a random series, Inst. Civil Eng. J. 4, 121 (1936).

[2] F. Garwood, An application of the theory of probability to the operation of vehicular-controlled traffic signals, J. Roy. Stat. Soc. (Supplement) 7, 65 (1940).

[3] M. S. Raff, The distribution of blocks in an uncongested stream of automobile traffic, J. Am. Stat. Soc. 46, 114 (1951).

[4] J. C. Tanner, The delay to pedestrians crossing a road, Biometrika 38, 383-342 (1951).

[5] A. J. Mayne, Some further results in the theory of pedestrians and road traffic, Biometrika 41, 375-389 (1954).

[6] G. H. Weiss, and A. A. Maradudin, Some problems in traffic delay, J. Op. Res. 10, 74 (1962).

[7] R. M. Oliver, and E. F. Bisbee, Queueing for gaps in high flow traffic streams, J. Op. Res. 10, 105 (1962).

[8] R. M. Oliver, Distribution of gaps and blocks in a traffic stream, J. Op. Res. 10, 197 (1962).

[9] W. S. Jewell, Waiting for a gap in traffic, Res. Report 6, Operations Research Center, University of California (Berkeley) (1961).

[10] J. Cohen, E. J. Dearnaley, and C. E. M. Hansel, The risk taken in crossing a road, Op. Res. Quart. 6, 120 (1955).

[11] P. A. Perchonok, and S. L. Levy, Applications of digital simulation techniques to freeway on-ramp traffic operations, Highway Research Board Proceedings, 506 (1960).

[12] R. Herman, and G. H. Weiss, Comments on the highway-crossing problem, J. Op. Res. 9, 828 (1961).

[13] W. L. Smith, Renewal theory and its ramifications, J. Roy. Stat. Soc. B20, 243 (1958).

[14] L. Takács, Introduction to the theory of queues, (Oxford Press, New York, 1962).

[15] W. S. Jewell, and R. M. Oliver, The distribution of spread, Res. Report 20, Operations Research Center, University of California (Berkeley) (1961).

(Paper 67B4-105) 